\title{
Chondroitin sulfate expression predicts poor outcome in breast cancer
}

\author{
KATRIN J. SVENSSON ${ }^{1}$, HELENA C. CHRISTIANSON ${ }^{1}$, PAULINA KUCHARZEWSKA ${ }^{1}$, VICTOR FAGERSTRÖM ${ }^{1}$, \\ LARS LUNDSTEDT $^{1}$, SIGNE BORGQUIST ${ }^{1}$, KARIN JIRSTRÖM ${ }^{2}$ and MATTIAS BELTING ${ }^{1,3}$ \\ ${ }^{1}$ Department of Clinical Sciences, Section of Oncology; ${ }^{2}$ Center for Molecular Pathology, Department \\ of Laboratory Medicine, Lund University, University and Regional Laboratories Region Skåne, \\ Malmö; ${ }^{3}$ University Hospital of Skåne, Department of Oncology, Lund, Sweden
}

Received June 2, 2011; Accepted July 11, 2011

DOI: 10.3892/ijo.2011.1164

\begin{abstract}
Experimental studies have established that the sulfated glycosaminoglycans heparan sulfate and chondroitin sulfate act as co-receptors of cytokines and growth factors that drive the malignant cell phenotype and the remodelling of the surrounding tumor stroma. However, the clinical relevance of these studies remains ill-defined. The present study investigates the significance of chondroitin sulfate expression in malignant cells and the stroma, respectively, of tumors from two independent cohorts of breast cancer patients (cohort I: 144 patients, 130 evaluable samples; cohort II: 498 patients, 469 evaluable samples; ER-positive patients $~ 86 \%$ in both cohorts). Kaplan-Meier analysis and Cox proportional hazards modelling were used to assess the relationship between chondroitin sulfate and recurrence-free and overall survival. High chondroitin sulfate expression in malignant cells was shown to predict shorter recurrence-free survival $(\mathrm{P}=0.007$, cohort $\mathrm{I}$; $\mathrm{P}=0.024$, cohort II) and overall survival (cohort $\mathrm{I}$ : $\mathrm{P}=0.044$; cohort II: $\mathrm{P}<0.001)$ in both cohorts. In multivariate analysis, high chondroitin sulfate in malignant cells was shown to be an independent, predictive factor of poor overall survival (cohort I: hazard ratio 2.28: $95 \%$ confidence interval 1.08-4.81, $\mathrm{P}=0.031$; cohort II: hazard ratio 1.71: $95 \%$ confidence interval 1.23-2.38, $\mathrm{P}=0.001)$. However, chondroitin sulfate in the stroma showed no correlation with known markers of tumor aggressiveness or with clinical outcome in either cohort. Our data suggest that high chondroitin sulfate expression in malignant cells is associated with an adverse outcome in patients with primary
\end{abstract}

Correspondence to: Dr Mattias Belting, Department of Clinical Sciences, Section of Oncology, Lund University, Barngatan 2B, SE-221 85, Lund, Sweden

E-mail: mattias.belting@med.lu.se

Abbreviations: CS, chondroitin sulfate; ER, estrogen receptor; GAG, glycosaminoglycan; HS, heparan sulfate; IHC, immunohistochemistry; MC, malignant cell; NHG, Nottingham histological grade; OS, overall survival; PG, proteoglycan; PR, progesterone receptor; RFS, recurrence-free survival; TGF- $\beta$, transforming growth factor $\beta$

Key words: breast cancer, chondroitin sulfate, proteoglycan breast cancer, supporting the idea of a functional and potentially targetable role of chondroitin sulfate in tumor disease.

\section{Introduction}

Recent advances in cancer research have clearly established that the development of cancer involves a complex interplay between transformed cancer cells and their surrounding stroma $(1,2)$. Studies aimed at the identification of prognostic biomarkers and treatment targets of cancer have, so far, focused on proteins typically expressed by malignant cells, while studies of typical stromal components e.g. glycoproteins remain relatively sparse. Notably, complex glycans lack a given template for their biosynthesis, which requires the concerted action of several, sometimes $>10$, different enzymes. This fact complicates the analysis at the genomic level of the potential role of glycoproteins in cancer.

The proteoglycans (PGs) constitute a large family of proteins characterized by the conjugation with sulfated glycosaminoglycan (GAG) polysaccharide chains. Experimental studies have suggested that sulfated GAGs have important roles in tumor development through interactions with major tumor promoting growth factors, cytokines and proteases $(3,4)$. Cell-surface PGs act as co-receptors of e.g. VEGF, FGF and PDGF-BB by presenting them to their respective high affinity, tyrosine kinase receptors. Extracellular matrix-resident $\mathrm{PGs}$ regulate the composition of the tumor microenvironment through interactions with proteases as well as collagens and glycoproteins in the tumor stroma. Also, matrix PGs serve as a reservoir for secreted factors, thereby shaping gradients of molecules involved in angiogenesis and tumor metastasis. PGs may thus regulate tumor development through direct effects in malignant cells and by shaping the architecture of the tumor stroma.

The involvement of sulphated GAGs of the heparan sulfate (HS) type in tumor biology has been extensively studied $(4,5)$. Despite their wide distribution and relative structural similarity to HS, chondrotin sulfate (CS) GAGs have attracted less attention. There is growing evidence that CS may be equally important in cancer disease by supporting the malignant behaviour of cancer cells and by promoting angiogenesis (6-10).

Ozzello et al (11) showed that adding CS to the culture medium supports the growth of breast cancer cells, and later 
studies revealed the expression of CS in breast cancer cells and malignant breast tumors (12-14). More recently, Suwiwat et al (15) reported that CS expression in the stroma of tumors from patients with node-negative breast cancer showed no correlation with clinical outcome. Interestingly, it was reported that in a small subset of the tumors, malignant cells expressed evident levels of CS; however, the clinical relevance of this finding was not further explored (15). The clinical role of CS in breast cancer thus remains to be determined. Here we have investigated how CS in the stroma and malignant cells, respectively, correlate with clinicopathological parameters and patient outcome in two independent cohorts of primary breast cancer.

\section{Materials and methods}

Antibodies and chemicals. CS-56 (C8035) mouse monoclonal anti-CS antibody was from Sigma, St. Louis, MO, Alexa Fluor488 goat anti-mouse antibody and phalloidin-TRITC were from Invitrogen. Cell media, supplements and chondroitinase ABC lyase (E.C. 4.2.2.4) were from Sigma, Sweden, biotinylated goat anti-mouse IgG secondary antibody (K 5007), Dako Envision kit K 5007, citrate buffer pH 6.0 (S 1699), Techmate 500Plus, peroxidase blocking solution (S 2023), Ki-67 antibody (M7240) were all from Dako, Denmark. Antibodies against estrogen receptor (ER; anti-ER, clone 6F11), progesterone receptor (PR; anti-PgR, clone 16) and human epidermal growth factor receptor 2 (HER2; Pathway CB-USA, 760-2694) were from Ventana Medical Systems Inc., AZ.

Cell culture and enzyme treatments. Human breast cancer cell lines MDA-MB-231 were purchased from the ATCC. Routine culture was performed in a humidified $5 \% \mathrm{CO}_{2}$ incubator at $37^{\circ} \mathrm{C}$ in RPMI medium supplemented with $10 \%$ foetal bovine serum (FBS), $2 \mathrm{mM}$ L-glutamine, $100 \mathrm{U} / \mathrm{ml}$ penicillin and $100 \mu \mathrm{g} / \mathrm{ml}$ streptomycin (growth medium). For CS digestion experiments, cells or tissue sections were incubated with digestion buffer [DMEM, $0.5 \%(\mathrm{w} / \mathrm{v})$ bovine serum albumin (BSA), and $20 \mathrm{mM}$ HEPES-HCl, pH 7.4] supplemented with $80 \mathrm{mU} / \mathrm{ml}$ chondroitinase $\mathrm{ABC}$ lyase for $2 \mathrm{~h}$, and additional $50 \mathrm{mU} / \mathrm{ml}$ for another $2 \mathrm{~h}$.

Flow cytometry and confocal laser scanning microscopy. Flow cytometry and confocal microscopy experiments were performed essentially as previously described $(16,17)$. For cell-surface CS staining, breast cancer cells were detached with PBS (2X) supplemented with $0.5 \mathrm{mM}$ EDTA, washed with PBS/BSA $1 \%(\mathrm{w} / \mathrm{v})$, and incubated with CS-56 antibody (titer 1:200) in PBS/BSA $1 \%$ for $30 \mathrm{~min}$ at $4^{\circ} \mathrm{C}$. Cells were then washed in PBS/BSA $1 \%$ and incubated with Alexa Fluor-488 conjugated goat anti-mouse antibody (1:200) for $30 \mathrm{~min}$ at $4^{\circ} \mathrm{C}$. Cells were washed in PBS/BSA $1 \%$ and analyzed by flow cytometry on a FACS-Calibur instrument integrated with Cell-Quest software (BD Biosciences).

For confocal microscopy experiments, subconfluent cells in 8-well chamber slides were washed with PBS and fixed with $2 \%$ paraformaldehyde for $5 \mathrm{~min}$ at RT, washed and blocked with PBS/BSA $1 \%$ for 30 min and stained with CS-56 antibody (1:200) at $4^{\circ} \mathrm{C}$ overnight. Cells were washed with PBS/BSA $1 \%$ and subsequently incubated with Alexa Fluor-488 goat anti-mouse antibody (1:200) for $30 \mathrm{~min}$ at RT, counterstained for f-actin using phalloidin-TRITC (1:500), and analyzed using Zeiss LSM 710 confocal scanning equipment with a 20X objective. Controls without primary antibody were included in all experiments, and data shown are representative of at least three independent experiments.

Patient characteristics. Tissue microarray (TMA) studies were performed on two separate patient cohorts. The screening cohort, cohort I, described elsewhere in detail (18) consisted of 144 cases of invasive breast cancer diagnosed at the Department of Pathology, Malmö University Hospital, Malmö, Sweden, between 2001 and 2002. The median age at diagnosis was 65 years (range 35-97), and the median follow-up time for overall survival (OS) was 52 months. Patients did not receive neoadjuvant therapy and were treated with either modified radical mastectomy or wide local excision. The median tumor size was $2.2 \mathrm{~cm} ; 67 \%$ of the tumors were PR-positive and $86 \%$ were ER-positive. Complete endocrine treatment data were available for 143 patients, 67 of whom received adjuvant tamoxifen, 3 an aromatase inhibitor, and 25 a combination of tamoxifen and an aromatase inhibitor. Information on adjuvant chemotherapy was available for 143 patients, of whom 30 received treatment. The second (validation) cohort, cohort II, included 498 patients with primary invasive breast cancer diagnosed at the Malmö University Hospital between 1988 and 1992. These cases belonged to an original cohort of 512 patients, as previously described in detail (19-21). The median age at diagnosis was 65 years, and median follow-up time to first breast cancer event was 128 months. Complete endocrine treatment data were available for 379 patients, 160 of whom received adjuvant tamoxifen. Information on adjuvant chemotherapy was available for 382 patients, of whom 23 received treatment. Information on the date of death was retrieved from regional cause-of-death registries for all patients in both cohorts. Ethical permission for the present investigation was obtained from the Lund University Regional Ethics Board, whereby informed consent was deemed not to be required other than by the opt-out method.

Tissue microarray construction and immunohistochemistry. Prior to TMA construction, all cases were histopathologically re-evaluated on hematoxylin- and eosin-stained slides by a senior pathologist (KJ). To assemble TMAs, defined areas of tumor tissue were indicated on a slide with a fresh tissue section from the paraffin block (22). Two biopsy samples, $1.0 \mathrm{~mm}$ in diameter, were taken from each donor paraffin block corresponding to the marked area using a manual arraying device (MTA-1, Beecher Inc, WI, USA). In general, cores were taken from the peripheral aspect of the tumor, and necrotic tissue was avoided. Expression of CS was determined using the Dako Envision kit K 5007 (an indirect polymer reinforcement technique). Consecutive sections of $4 \mu \mathrm{m}$ in diameter were dried, deparaffinized, rehydrated and subjected to heat-induced antigen retrieval in citrate buffer, $\mathrm{pH}$ 6.0. Tissue sections were blocked in peroxidase blocking solution for $10 \mathrm{~min}$, followed by incubation with a monoclonal mouse anti-CS antibody (CS-56), diluted at 1:200 for $30 \mathrm{~min}$ at room temperature in a Techmate 500Plus. Subsequently, biotinylated goat anti-mouse IgG secondary antibody was applied to the sections, followed by peroxidase-conjugated streptavidin and DAB for visualiza- 
tion. For all other antibodies, heat-induced antigen retrieval in citrate buffer, $\mathrm{pH}$ 6.0, was performed prior to processing of slides in the Ventana Benchmark system (Ventana Medical Systems Inc., AZ) using pre-diluted antibodies to the ER, PR and human epidermal growth factor receptor HER2, or in the Dako Techmate 500 system for Ki-67.

Evaluation of staining. Following antibody optimization and staining, the expression of CS was evaluated in 130 (90\%) of the 144 tumors in cohort I, and 469 (94\%) of the 498 tumors in cohort II. The missing tumors were not available for analysis due to core loss during sectioning and staining. Malignant cellassociated CS and stromal CS staining were assessed separately. The geographic limit for assessment of tumor stroma staining was defined as areas surrounded by neoplastic cells. Scoring was performed according to staining intensity on a scale as follows: 0 , negative; 1 , weak; 2 , moderate; and 3 , strong intensity. ER, PR, HER2 and Ki-67 were assessed as previously described (23). Definitions of ER and PR negativity followed current clinical guidelines ( $<10 \%$ positive nuclei). IHC staining was evaluated by an observer who was blinded to clinical and outcome data.

Statistical analysis. Differences in distribution of pathological and clinical parameters (age, tumor size, histological grade, lymph node status, hormone receptor status, HER2 and Ki-67) between CS-low (staining intensities 0+1) and CS-high (staining intensities 2+3) in malignant cells and stroma, respectively, were calculated by Chi-square test for categorized variables and Chi-square test for trend of variables with more than two categories. Kaplan-Meier analysis and log-rank test were used to illustrate differences between recurrence-free survival (RFS) and overall survival (OS) according to CS expression. Cox regression proportional hazards models were used to estimate the impact of CS expression on RFS and OS in both univariate and multivariate analyses, adjusted for tumour size, ER, HER2, lymph node status and Nottingham histological grade $(\mathrm{NHG})$ of the entire cohorts. All statistical tests were two-sided and $\mathrm{P}<0.05$ was considered statistically significant. Calculations were performed using SPSS, version 15.0 (SPSS Inc., Chicago, IL).

\section{Results}

CS expression in breast cancer cells and breast tumors. The epitope of the anti-CS antibody, CS-56, used in the present study was recently characterized, using an array of structurally defined, natural oligosaccharides (24). We found that the abundance of the CS-56 epitope could be found both in malignant cells (MCs) and in the tumor stroma (TS) of patient breast tumors, while absent in normal ductal epithelial cells. In Fig. 1, examples of tumors with different patterns of low and high CS staining intensity in MCs and TS are visualized. The CS-56 antibody epitope was also ubiquitously expressed in MDA-MB 231 cells, i.e. a well-characterized, highly malignant, breast cancer cell-line established from a patient tumor (Fig. 2B); enzymatic treatment of patient tumors or MDA-MB 231 breast cancer cells with chondroitinase ABC lyase that specifically degrades CS, substantially reduced CS-56 antibody staining thus validating the specificity of the antibody (Fig. 2B, C, and D).

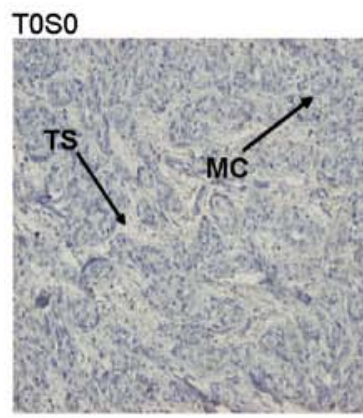

T1S2
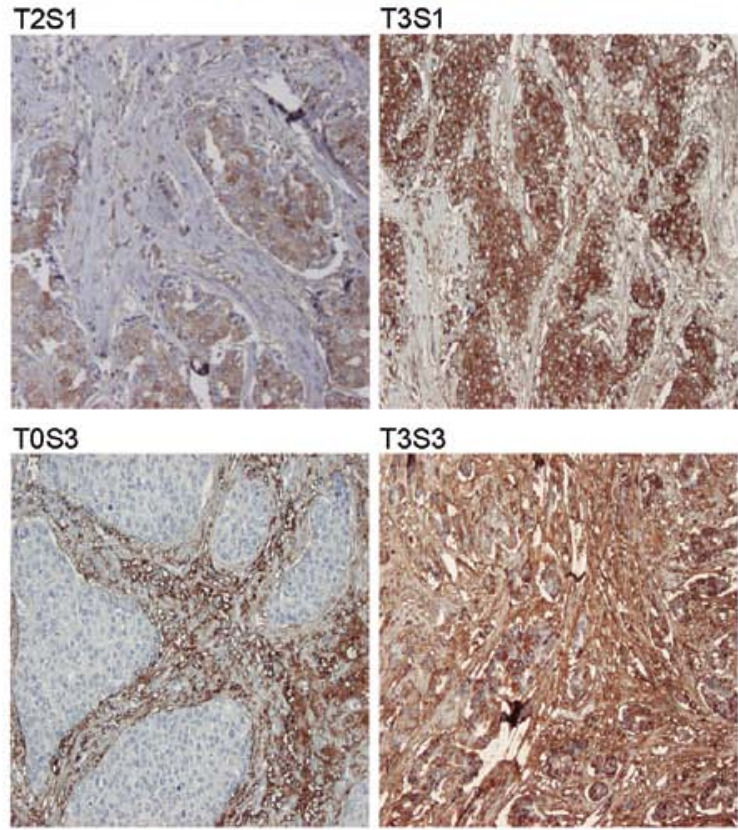

T3S3
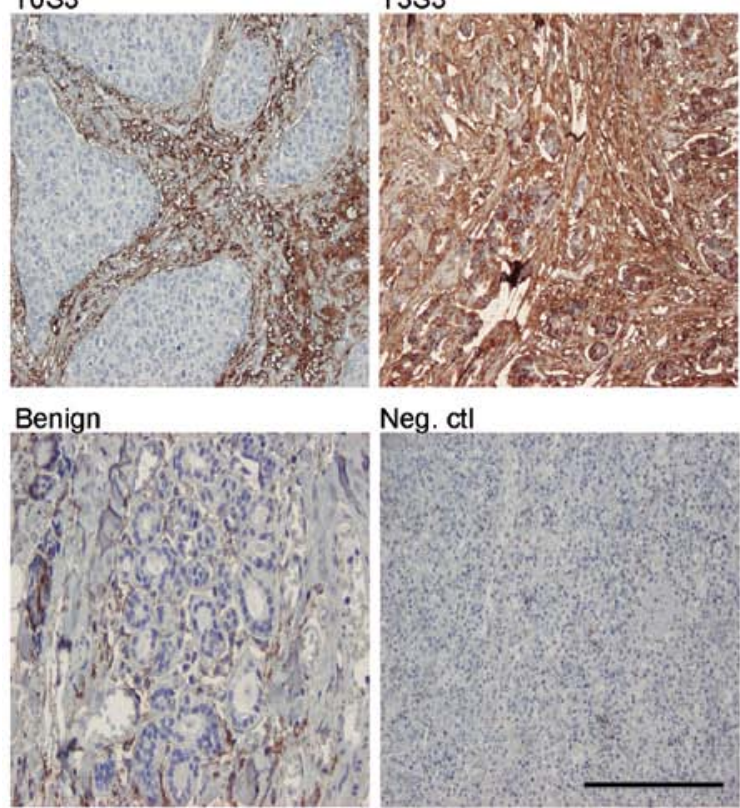

Neg. ctl

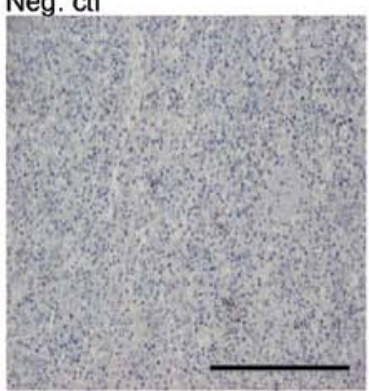

Figure 1. Variations in CS expression in malignant cells and the stroma of primary breast cancer. Representative images of CS expression in malignant cells (MC) and in the tumor stroma (TS). Shown are grade 0,1,2 and 3 stainings of malignant cells of the tumor (T) and the stroma (S), as indicated; low CS expression in normal breast epithelial cells (benign); negative control staining in the absence of primary antibody (Neg. ctl). Scale bar, $100 \mu \mathrm{m}$

High CS in malignant cells correlates with the aggressiveness of breast tumors. We next analyzed the clinical significance of CS expression in patient breast tumors. Initially, CS expression was analyzed by immunohistochemistry in a screening material of 130 breast cancer patients (cohort I). In some cases, CS staining of MCs was accentuated towards the membrane, whereas in others it was more diffusely distributed in the cytoplasm (data not shown). Different subcellular localizations were, however, not accounted for in the further analyses. Out of 130 evaluable samples, 44 and 117 stained positive (intensity scores 1-3) in MCs (34\%) and stroma (90\%), respectively. Moreover, 
A

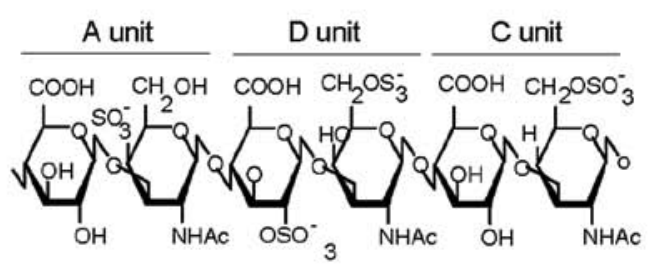

B

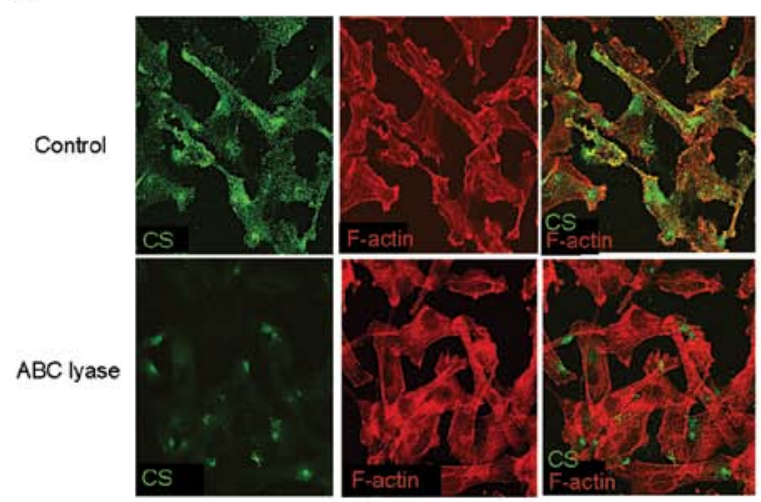

C

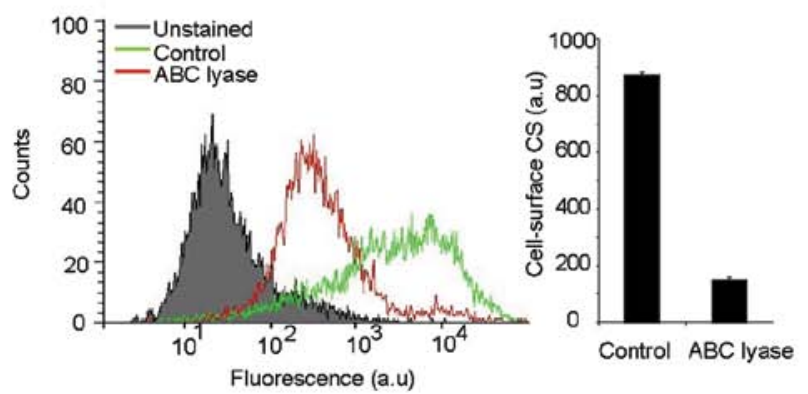

D

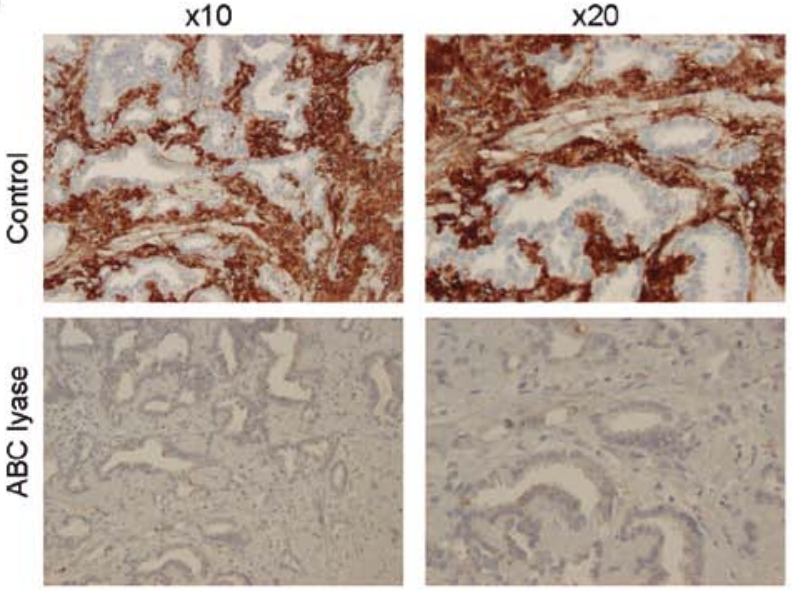

Figure 2. Expression of the CS-56 antibody epitope in breast cancer cells. (A) The major forms of CS include A-units [GlcUAb1-3GalNAc(4S)], C-units [GlcUAb1-3GalNAc(6S)], D-units [GlcUA(2S)b1-3GalNAc(6S)] and E-units [GlcUAb1-3GalNAc(4S,6S)], where $2 \mathrm{~S}, 4 \mathrm{~S}$ and $6 \mathrm{~S}$ designate 2-O-, 4-O-, and 6-O-sulfate groups, respectively. Shown is the CS epitope recognized by the CS-56 antibody. (B) MDA-MB-231 cells express significant levels of the CS-56 epitope. CS-56 antibody specificity was supported by substantial reduction of cell-surface staining after treatment with chondroitinase $A B C$ lyase that specifically degrades CS (lower panel). (C) Quantification by flow cytometry analysis showed an $\sim 85 \%$ reduction in cell-surface staining with CS-56 antibody in chondroitinase $\mathrm{ABC}$ lyase treated as compared with untreated MDA-MB-231 cells. Chondroitinase ABC lyase specifically cleaves CS polysaccharides. Right panel: Results are presented as the mean from at least two experiments, each performed in triplicate. (D) Substantial reduction of breast tumor staining with CS-56 antibody by treatment with chondroitinase ABC lyase, shown at lower (left panel) and higher (right panel) magnifications. about half (48\%) of the cases, showed strong stromal CS staining (intensity scores 2-3). To further determine the clinical significance of CS staining separately in MCs and TS, intensity scores were dichotomized into low $(0+1)$ and high $(2+3)$. In cohort I, high MC-CS showed a tendency, however not significant, towards an association with larger tumors $(\mathrm{P}=0.061)$, and correlated significantly with HER2 status $(\mathrm{P}=0.04)$ (Table I). Notably, there was no correlation between TS-CS and any relevant clinicopathological parameter in cohort I (data not shown).

CS staining and similar analyses were then performed in independent breast cancer patient material encompassing 469 evaluable tumors (cohort II). Again, there was no correlation of TS-CS with clinicopathological parameters (data not shown), whereas high MC-CS, similar to cohort I, showed a nonsignificant trend towards an association with larger tumor size $(\mathrm{P}=0.083)$; a similar relationship was found with histological grade, NHG $(\mathrm{P}=0.089)$. In this larger cohort, high MC-CS correlated significantly with the occurrence of lymph node metastasis $(\mathrm{P}=0.008)$ (Table I).

Together, the above data point towards a more aggressive phenotype in tumors displaying high CS expression specifically in MCs. Interestingly, Kaplan-Meier analysis showed that high MC-CS was associated with a significantly shorter recurrence-free survival (RFS) and overall survival (OS) in both cohorts (Fig. 3). This was confirmed by Cox univariate analysis where a high MC-CS correlated significantly with shorter RFS in both cohorts (cohort I: hazard ratio, 2.98: 95\% confidence interval, 1.35-6.58, $\mathrm{P}=0.007$; cohort II: hazard ratio, 1.59: $95 \%$ confidence interval, 1.06-2.39, $\mathrm{P}=0.024$ ), and similar associations were found between MC-CS and OS (Table II). The prognostic value of MC-CS was similar when evaluated in strata according to high and low stromal CS expression in both cohorts (data not shown). Moreover, the correlation between high MC-CS and patient outcome remained significant in the ER-positive subgroup, whereas no significant relationship was found in patients with ER-negative tumors (data not shown). These data are most likely explained by the strong predominance of ER-positive tumors ( 86\%) in both cohorts. Finally, multivariate analyses showed that high MC-CS is an independent marker of poor OS in both cohorts (cohort I, hazard ratio 2.28: 95\% confidence interval 1.08-4.81, $\mathrm{P}=0.031$; cohort II, hazard ratio 1.71: $95 \%$ confidence interval $1.23-2.38, \mathrm{P}=0.001$ ).

\section{Discussion}

Our data suggest that CS expression correlates with poor clinical outcome in primary breast cancer. This was true specifically for CS expression in malignant cells, whereas we found no such correlation for CS expressed in the stromal compartment. To our knowledge, this is the first report showing a correlative role of malignant cell expression of CS or any other type of sulfated GAG in patient tumors.

The role of CSPG core proteins in cancer has, however, been extensively studied, both experimentally and in clinical tumor specimens from various cancer types (15,25-34). In breast cancer, versican, i.e. an extracellular matrix protein substituted with CS chains at several positions, was found absent in malignant cells, whereas elevated stromal expression of versican core protein predicted a poor prognosis $(15,30)$. In contrast, reduced stromal expression of decorin, i.e. the prototype member of 
Table I. Correlation between CS expression in malignant cells and clinicopathological parameters in breast cancer cohorts I and II.

\begin{tabular}{|c|c|c|c|c|c|c|}
\hline \multirow{3}{*}{ Prognostic factor } & \multicolumn{6}{|c|}{ CS expression in malignant cells } \\
\hline & \multicolumn{3}{|c|}{ Cohort I } & \multicolumn{3}{|c|}{ Cohort II } \\
\hline & & & p-value & & & p-value \\
\hline CS intensity & $0-1$ & $2-3$ & & $0-1$ & $2-3$ & \\
\hline $\mathrm{n}$ & 103 & 27 & & 392 & 77 & \\
\hline \multicolumn{7}{|l|}{ Age } \\
\hline Median & 67 & 64 & 0.961 & 64 & 67 & 0.077 \\
\hline Range & $34-97$ & $49-88$ & & $27-96$ & $28-90$ & \\
\hline \multicolumn{7}{|l|}{ Size } \\
\hline Median & 20 & 25 & 0.061 & 17 & 19 & 0.083 \\
\hline Range & $7-145$ & $13-140$ & & $1-100$ & $1-100$ & \\
\hline \multicolumn{7}{|l|}{ NHG } \\
\hline I & 15 & 4 & 0.260 & 104 & 11 & 0.089 \\
\hline II & 42 & 15 & & 157 & 37 & \\
\hline II & 46 & 8 & & 130 & 20 & \\
\hline \multicolumn{7}{|l|}{ Nodal status } \\
\hline 0 & 54 & 10 & 0.152 & 232 & 34 & 0.008 \\
\hline 1 & 39 & 14 & & 119 & 35 & \\
\hline Missing & 10 & 3 & & & & \\
\hline \multicolumn{7}{|l|}{ ER status } \\
\hline Negative & 13 & 4 & 0.760 & 61 & 7 & 0.141 \\
\hline Positive & 90 & 23 & & 331 & 70 & \\
\hline \multicolumn{7}{|l|}{ PR status } \\
\hline Negative & 34 & 8 & 0.740 & 128 & 23 & 0.633 \\
\hline Positive & 69 & 19 & & 264 & 54 & \\
\hline \multicolumn{7}{|l|}{ Ki67 } \\
\hline $0-10 \%$ & 5 & 0 & 0.581 & 137 & 30 & 0.339 \\
\hline $11-25 \%$ & 33 & 13 & & 122 & 25 & \\
\hline$>25 \%$ & 51 & 12 & & 198 & 17 & \\
\hline \multicolumn{7}{|l|}{ HER 2} \\
\hline 0 & 96 & 23 & 0.040 & 347 & 71 & 0.701 \\
\hline 1 & 4 & 4 & & 35 & 6 & \\
\hline
\end{tabular}

ER, estrogen receptor; PR, progesterone receptor; HER2, human epidermal growth factor 2; NHG, Nottingham histological grade. Ten \% cutoff used for determination of hormone receptor status. Mann-Whitney U-test for comparison of medians and Chi-square test for X x 2 tables. The category marked missing was not included in the analysis.

the small leucine-rich CSPGs, was associated with a worse prognosis of breast cancer (31). Similar relationships between stromal expression of versican and decorin and clinical outcome were reported for prostate cancer (29). Staining for CS with the CS-56 antibody showed a significant correlation between elevated stromal CS and poor prognosis in prostate cancer (28). In line with the results of the present investigation, no significant correlation between stromal CS expression and clinical outcome was found in a relatively small cohort $(n=86)$ of node-negative breast cancer (15), implying that the prognostic roles of stromal expression of PG core proteins and CS chains, respectively, are not concordant between tumor types.

More recently, it was suggested that down-regulation in malignant cells of syndecan-1, i.e. a plasma membrane inte- grated protein substituted with CS and/or HS, and concomitant induction of stromal syndecan-1 expression is associated with epithelial-mesenchymal transition and, accordingly, worse prognosis of breast cancer (32). It is currently unknown which CSPG core protein/s is specifically associated with CS chains harbouring the CS-56 antibody epitope, and whether increased tumor aggressiveness is related to altered expression and/or degree of glycosylation of a particular PG in malignant cells. However, previous data altogether point to the involvement of PGs other than versican and syndecan-1.

A remaining key question is which signalling pathway dictate the expression level and structure of CS in breast cancer tissue. The biological function of most GAGs is determined by their sulfation pattern, providing the polysaccharide chain 
A
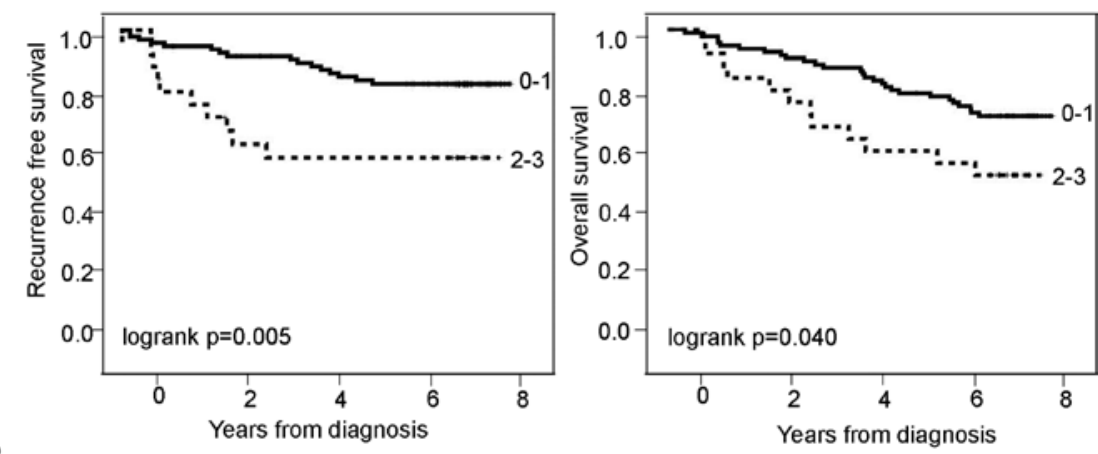

B
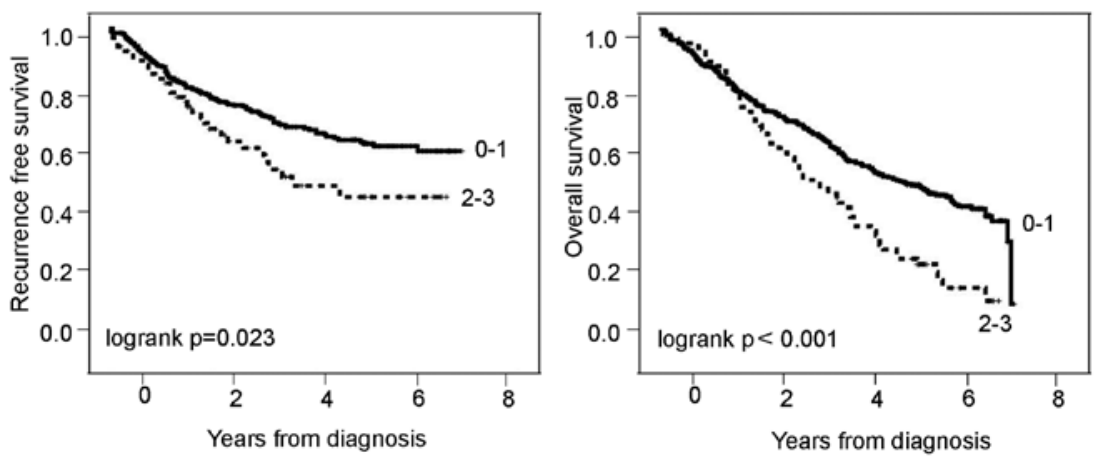

Figure 3. CS expression in malignant cells correlates with tumor aggressiveness. Kaplan-Meier estimates of recurrence-free survival (left panels) and overall survival (right panels) according to low (0-1) versus high (2-3) CS expression in malignant cells of tumors from cohort I (A) and cohort II (B). In both cohorts, high CS expression in malignant cells was significantly associated with shorter recurrence-free survival and overall survival.

Table II. Cox regression analyses of recurrence-free and overall survival according to CS expression (dichtomized 0-1 vs. 2-3).

\begin{tabular}{|c|c|c|c|c|c|c|}
\hline & \multicolumn{3}{|c|}{ Cohort I } & \multicolumn{3}{|c|}{ Cohort II } \\
\hline & $\mathrm{n}$ & $\mathrm{RR}(95 \% \mathrm{CI})$ & p-value & $\mathrm{n}$ & RR $(95 \% \mathrm{CI})$ & $\mathrm{p}$-value \\
\hline \multicolumn{7}{|c|}{ Univariate recurrence-free survival } \\
\hline $0-1$ & $(n=103)$ & 1.00 & & $(n=392)$ & 1.00 & \\
\hline $2-3$ & $(\mathrm{n}=27)$ & $2.98(1-35-6.58)$ & 0.007 & $(\mathrm{n}=77)$ & $1.59(1.06-2.39)$ & 0.024 \\
\hline \multicolumn{7}{|c|}{ Multivariate recurrence-free survival } \\
\hline $0-1$ & $(n=103)$ & 1.00 & & $(n=392)$ & 1.00 & \\
\hline $2-3$ & $(n=27)$ & $2.11(0.81-5.49)$ & 0.125 & $(n=77)$ & $1.36(0.89-2.09)$ & 0.157 \\
\hline \multicolumn{7}{|c|}{ Univariate overall survival } \\
\hline $0-1$ & $(n=103)$ & 1.00 & & $(n=392)$ & 1.00 & \\
\hline $2-3$ & $(\mathrm{n}=27)$ & $2.01(1.02-3.97)$ & 0.044 & $(\mathrm{n}=77)$ & $1.73(1.28-2.34)$ & $<0.001$ \\
\hline \multicolumn{7}{|c|}{ Multivariate overall survival } \\
\hline $0-1$ & $(n=103)$ & 1.00 & & $(n=392)$ & 1.00 & \\
\hline $2-3$ & $(\mathrm{n}=27)$ & $2.28(1.08-4.81)$ & 0.031 & $(\mathrm{n}=77)$ & $1.71(1.23-2.38)$ & 0.001 \\
\hline
\end{tabular}

Multivariate analysis included adjustment for age (continuous), nodal status (neg/pos), NHG (I-II vs III), size (continuous), ER (cut-off 10\%) and HER 2 (0-2 vs 3). All parameters except HER2 were prognostic in univariate analysis in both cohorts. HER2 was only prognostic in cohort I and was therefore not included in the multivariate analysis for cohort II.

with a high negative charge and high affinity interactions with growth factors, cytokines, enzymes and matrix proteins of the tumor microenvironment (3-6). The formation of the CS-56 antibody epitope (Fig. 2A), requires the concerted action of three separate sulfotransferases during CS biosynthesis, i.e. GalNAc 4-O-sulfotransferase, GalNac 6-O-sulfotransferase, and GlcUA 2-O-sulfotransferase. In addition, at least another five enzymes are required to build the CS polymer. Our data 
may reflect the induction of one or several CS polymerizing enzymes or sulfotransferases in aggressive breast cancer cells. This question can potentially be addressed at the transcriptome level by analysis of relevant glycosyltransferase and sulfotransferase enzymes in tumors expressing different levels of CS.

There is growing evidence for a major role of transforming growth factor $\beta$ (TGF- $\beta$ ) in the propagation of epithelial-mesenchymal transition and the progression of breast cancer (35-37). In the context of the present investigation, it is of interest that TGF- $\beta$ has been shown to induce CS in several types of cells, e.g. in mammary epithelial cells (38), melanoma cells (39) and in human lung fibroblasts (40). At a mechanistic level, TGF- $\beta$ was shown to up-regulate xylosyltransferase-1, i.e. a rate-limiting enzyme that initiates $\mathrm{CS}$ attachment onto $\mathrm{PG}$ core protein $(41)$ and GalNAc 4-O-sulfotransferase $(42,43)$ that catalyzes one of the key steps in CS-56 epitope formation. Our group is currently investigating whether CS induction constitutes an important down-stream event of the TGF- $\beta$ signalling pathway during epithelial-mesenchymal transition and cancer progression.

Experimental studies suggest that CS has a functional role in breast cancer, and that targeting of CS may represent a therapeutic strategy in cancer treatment (44), e.g. CS derivatives potently reduced the viability of breast cancer cells, and inhibited tumor growth as well as hematogenous metastasis in experimental models $(45,46)$. Several clinical studies indicate that a CS-related GAG, heparin, independently of its anti-thrombotic activity has direct tumor inhibiting effects, and ongoing phase III clinical studies aim at validating these findings $(47,48)$. CS may thus be an attractive alternative to heparin given its inhibitory activities on tumor growth, angiogenesis and metastasis, without undesirable anti-coagulant side effects. In summary, our data indicate that malignant cell expression of CS correlates with clinical outcome of breast cancer patients, which supports a functional and potentially targetable role of CS in tumor disease.

\section{Acknowledgements}

We are grateful to Kristina Lövgren for expert technical assistance and to Dr Mårten Fernö for critical reading of the manuscript. This work was supported by grants from the Swedish Cancer Society, the Swedish Research Council, the Swedish Society of Medicine, the Crafoordska, Gunnar Nilsson, Lundbergs, and Kamprad Foundations, the Lund University Hospital donation funds, the Medical Faculty (Lund University), and the Governmental funding of clinical research within the national health services (ALF).

\section{References}

1. Heldin $\mathrm{CH}$, Rubin K, Pietras K and Ostman A: High interstitial fluid pressure - an obstacle in cancer therapy. Nat Rev Cancer 10: 806-813, 2004.

2. Radisky DC, Stallings-Mann M, Hirai Y and Bissell MJ: Single proteins might have dual but related functions in intracellular and extracellular microenvironments. Nat Rev Mol Cell Biol 3: 228-234, 2009.

3. Belting M: Heparan sulfate proteoglycan as a plasma membrane carrier. Trends Biochem Sci 28: 145-151, 2003.

4. Fuster MM and Esko JD: The sweet and sour of cancer: glycans as novel therapeutic targets. Nat Rev Cancer 5: 526-542, 2005.
5. Jakobsson L, Kreuger J, Holmborn K, et al: Heparan sulfate in trans potentiates VEGFR-mediated angiogenesis. Dev Cell 10: 625-634, 2006

6. Malavaki C, Mizumoto S, Karamanos N and Sugahara K: Recent advances in the structural study of functional chondroitin sulfate and dermatan sulfate in health and disease. Connect Tissue Res 49: 133-139, 2008.

7. Wegrowski $\mathrm{Y}$ and Maquart FX: Chondroitin sulfate proteoglycans in tumor progression. Adv Pharmacol 53: 297-321, 2006.

8. Li F, Ten Dam GB, Murugan S, et al: Involvement of highly sulfated chondroitin sulfate in the metastasis of the Lewis lung carcinoma cells. J Biol Chem 283: 34294-34304, 2008.

9. Sugahara KN, Hirata T, Tanaka T, et al: Chondroitin sulfate fragments enhance CD44 cleavage and CD44-dependent motility in tumor cells. Cancer Res 68: 7191-7199, 2008.

10. Cortes M, Baria AT and Schwartz NB: Sulfation of chondroitin sulfate proteoglycans is necessary for proper Indian hedgehog signaling in the developing growth plate. Development 136: 1697-1706, 2009

11. Ozzello L, Lasfargues EY and Murray MR: Growth-promoting activity of acid mucopolysaccharides on a strain of human mammary carcinoma cells. Cancer Res 20: 600-604, 1960.

12. Chandrasekaran EV and Davidson EA: Glycosaminoglycans of normal and malignant cultured human mammary cells. Cancer Res 39: 870-880, 1979.

13. Gowda DC, Bhavanandan VP and Davidson EA: Isolation and characterization of proteoglycans secreted by normal and malignant human mammary epithelial cells. J Biol Chem 261: 4926-4934, 1986.

14. Alini M and Losa GA: Partial characterization of proteoglycans isolated from neoplastic and nonneoplastic human breast tissues. Cancer Res 51: 1443-1447, 1991.

15. Suwiwat S, Ricciardelli C, Tammi R, et al: Expression of extracellular matrix components versican, chondroitin sulfate, tenascin, and hyaluronan, and their association with disease outcome in node-negative breast cancer. Clin Cancer Res 10: 2491-2498, 2004

16. Welch JE, Bengtson P, Svensson K, et al: Single chain fragment anti-heparan sulfate antibody targets the polyamine transport system and attenuates polyamine-dependent cell proliferation. Int J Oncol 32: 749-756, 2008.

17. Kucharzewska P, Welch JE, Svensson KJ and Belting M: Ornithine decarboxylase and extracellular polyamines regulate microvascular sprouting and actin cytoskeleton dynamics in endothelial cells. Exp Cell Res 16: 2683-91, 2010.

18. Brennan DJ, Rexhepaj E, O'Brien SL, et al: Altered cytoplasmicto-nuclear ratio of survivin is a prognostic indicator in breast cancer. Clin Cancer Res 14: 2681-2689, 2008.

19. Borgquist S, Jögi A, Pontén F, Rydén L, Brennan DJ and Jirström K: Prognostic impact of tumour-specific HMG-CoA reductase expression in primary breast cancer. Breast Cancer Res 10: R79, 2008.

20. O'Brien SL, Fagan A, Fox EJ, et al: CENP-F expression is associated with poor prognosis and chromosomal instability in patients with primary breast cancer. Int J Cancer 120: 1434-1443, 2007.

21. Paulsson J, Sjoblom T, Micke P, et al: Prognostic significance of stromal platelet-derived growth factor beta receptor expression in human breast cancer. Am J Pathol 175: 334-341, 2009.

22. Kononen J, Bubendorf L, Kallionimeni A, et al: Tissue microarrays for high-throughput molecular profiling of tumor specimens. Nat Med 4: 844-847, 1998.

23. Borgquist S, Holm C, Stendahl M, Anagnostaki L, Landberg G and Jirstrom K: Oestrogen receptors alpha and beta show different associations to clinicopathological parameters and their coexpression might predict a better response to endocrine treatment in breast cancer. J Clin Pathol 61: 197-203, 2008.

24. Ito Y, Hikino M, Yajima Y, et al: Structural characterization of the epitopes of the monoclonal antibodies 473HD, CS-56, and MO-225 specific for chondroitin sulfate D-type using the oligosaccharide library. Glycobiology 15: 593-603, 2005.

25. Adany R, Heimer R, Caterson B, Sorrell JM and Iozzo RV: Altered expression of chondroitin sulfate proteoglycan in the stroma of colon cancer. J Biol Chem 265: 1389-1396, 1990.

26. Cohen IR, Murdoch AD, Naso MF, Marchetti D, Berd D and Iozzo RV: Abnormal expression of perlecan proteoglycan in metastatic melanomas. Cancer Res 54: 5771-5774, 1994.

27. Santra M, Skorski T, Calabretta TB, Lattime EC and Iozzo RV: De novo decorin gene expression suppresses the malignant phenotype in human colon cancer cells. Proc Natl Acad Sci USA 92: 7016-7020, 1995 
28. Ricciardelli C, Mayne K, Sykes PJ, et al: Elevated stromal chondroitin sulfate glycosaminoglycan predicts progression in early-stage prostate cancer. Clin Cancer Res 3: 983-992, 1997.

29. Ricciardelli C, Mayne K, Sykes PJ, et al: Elevated levels of versican but not decorin predict disease progression in earlystage prostate cancer. Clin Cancer Res 4: 963-971, 1998.

30. Ricciardelli C, Brooks JH, Suwiwat S, et al: Regulation of stromal versican expression by breast cancer cells and importance to relapse-free survival in patients with node-negative primary breast cancer. Clin Cancer Res 8: 1054-1060, 2002.

31. Troup S, Njue C, Kliewer EV, et al: Reduced expression of the small leucine-rich proteoglycans, lumican, and decorin is associated with poor outcome in node-negative invasive breast cancer. Clin Cancer Res 9: 207-214, 2003.

32. Loussouarn D, Campion L, Sagan C, et al: Prognostic impact of syndecan-1 expression in invasive ductal breast carcinomas. $\mathrm{Br} \mathbf{J}$ Cancer 98: 1993-1998, 2008.

33. Goldoni S and Iozzo RV: Tumor microenvironment: Modulation by decorin and related molecules harboring leucine-rich tandem motifs. Int J Cancer 123: 2473-2479, 2008.

34. Ricciardelli C, Sakko AJ, Ween MP, Russell DL and Horsfall DJ: The biological role and regulation of versican levels in cancer. Cancer Metastasis Rev 28: 233-245, 2009.

35. Muraoka RS, Dumont N, Ritter CA, et al: Blockade of TGF-beta inhibits mammary tumor cell viability, migration, and metastases. J Clin Invest 109: 1551-1559, 2002.

36. Vincent T, Neve EP, Johnson JR, et al: A SNAIL1-SMAD3/4 transcriptional repressor complex promotes TGF-beta mediated epithelial-mesenchymal transition. Nat Cell Biol 11: 943-950, 2009.

37. Desruisseau S, Palmari J, Giusti C, Romain S, Martin PM and Berthois Y: Determination of TGFbetal protein level in human primary breast cancers and its relationship with survival. Br J Cancer 94: 239-246, 2006.

38. Rapraeger A: Transforming growth factor (type beta) promotes the addition of chondroitin sulfate chains to the cell surface proteoglycan (syndecan) of mouse mammary epithelia. J Cell Biol 109: 2509-2518, 1989.

39. Heredia A, Villena J, Romarís M, Molist A and Bassols A Transforming growth factor beta 1 increases the synthesis and shedding of the melanoma-specific proteoglycan in human melanoma cells. Arch Biochem Biophys 333: 198-206, 1996.
40. Romarís M, Bassols A and David G: Effect of transforming growth factor-beta 1 and basic fibroblast growth factor on the expression of cell surface proteoglycans in human lung fibroblasts. Enhanced glycanation and fibronectin-binding of CD44 proteoglycan, and down-regulation of glypican. Biochem J 310: 73-81, 1995

41. Prante C, Milting H, Kassner A, et al: Transforming growth factor beta1-regulated xylosyltransferase I activity in human cardiac fibroblasts and its impact for myocardial remodeling. J Biol Chem 282: 26441-26449, 2007.

42. Tiedemann K, Olander B, Eklund E, et al: Regulation of the chondroitin/dermatan fine structure by transforming growth factor-beta1 through effects on polymer-modifying enzymes. Glycobiology 15: 1277-1285, 2005.

43. Willis CM, Wrana JL and Klüppel M: Identification and characterization of TGFbeta-dependent and -independent cisregulatory modules in the C4ST-1/CHST11 locus. Genet Mol Res 8: 1331-1343, 2009.

44. Yamada S and Sugahara K: Potential therapeutic application of chondroitin sulfate/dermatan sulfate. Curr Drug Discov Technol 5: 289-301, 2008.

45. Pumphrey CY, Theus AM, Li S, Parrish RS and Sanderson RD: Neoglycans, carbodiimide-modified glycosaminoglycans: a new class of anticancer agents that inhibit cancer cell proliferation and induce apoptosis. Cancer Res 62: 3722-3728, 2002.

46. Borsig L, Wang L, Cavalcante MC, et al: Selectin blocking activity of a fucosylated chondroitin sulfate glycosaminoglycan from sea cucumber. Effect on tumor metastasis and neutrophil recruitment. J Biol Chem 282: 14984-14991, 2007.

47. Altinbas M, Coskun HS, Er O, et al: A randomized clinical trial of combination chemotherapy with and without low-molecularweight heparin in small cell lung cancer. $\mathrm{J}$ Thromb Haemost 2: 1266-1271, 2004

48. Klerk C, Smorenburg SM, Otten HM, et al: The effect of LMWH on survival in patients with advanced malignancy. J Clin Oncol 23: 2130-2135, 2005. 